\title{
Increased expression of mRNA for matrix metalloproteinases- 1 and -3 and tissue inhibitor of metalloproteinases- 1 in intestinal biopsy specimens from patients with coeliac disease
}

\author{
S Daum, U Bauer, H-D Foss, D Schuppan, H Stein, E-O Riecken, R Ullrich
}

\begin{abstract}
Background-Extracellular matrix (ECM) degradation may play a role in villus atrophy in coeliac disease (CD).

Aims-To compare the cellular expression of mRNA transcripts for the two major matrix degrading proteases, matrix metalloproteinase (MMP)-1 and MMP-3, their inhibitor, tissue inhibitor of metalloproteinases (TIMP)-1, and procollagen I in the intestinal mucosa of patients with untreated and treated $C D$ and normal controls.
\end{abstract}

Patients/Methods-Duodenal biopsy specimens from ten untreated CD patients, from six of these after a gluten free diet, and from ten control patients were hybridised with ${ }^{35} \mathrm{~S}$-labelled RNA probes. The number of positive cells in the subepithelial region and lamina propria were counted microscopically.

Results-The numbers of cells positive for MMP-1 ( $<<0.005)$, MMP-3 $(p<0.01)$, and TIMP-1 $(p<0.05)$ mRNA were higher in the subepithelial region of $C D$ mucosa than in that from controls. In the lamina propria, only cells positive for MMP-1 mRNA were increased in CD patients compared with controls $(p<0.01)$. MMP-1 and MMP-3 mRNA expression returned to normal in CD patients after treatment with a gluten free diet $(p<0.05)$, while TIMP-1 mRNA expression remained elevated. The number of procollagen I mRNA expressing cells did not change. Expression of MMP-1 and MMP-3 MRNA was mainly localised to subepithelial fibroblasts and macrophages.

Conclusions-The decreased ratio of collagen I and TIMP-1 mRNA expressing cells to MMP-1 and MMP-3 mRNA expressing cells in untreated $C D$ suggests a shift towards ECM degradation. ECM degradation by activated subepithelial fibroblasts and macrophages may be an important mechanism driving mucosal transformation in CD.

(Gut 1999;44:17-25)

Keywords: coeliac disease; extracellular matrix; villus atrophy; matrix metalloproteinases; TIMP-1; collagen I

Coeliac disease (CD) is a gluten sensitive enteropathy characterised by deepening of the crypts and flattening of the villi. ${ }^{1}$ Infiltration of the mucosal wall with activated lymphocytes, neutrophils, plasma cells, and eosinophils can be demonstrated, but the pathogenesis of CD is still unclear. ${ }^{12}$

The remarkable increase in intraepithelial $\mathrm{T}$ cells and the isolation of gluten responsive $T$ cell clones from intestinal biopsy samples from patients with CD suggests a central role for $\mathrm{T}$ cells in the immunopathogenesis of $\mathrm{CD}^{3} \mathrm{~T}$ cells can induce hyper-regenerative mucosal transformation-that is, villus atrophy and hyperplasia of the crypts - as shown by direct activation of $\mathrm{T}$ cells in explanted fetal gut mucosa. ${ }^{4} \quad$ Interleukin-1, interferon- $\gamma$, interferon $-\alpha / \beta$, and tumour necrosis factor- $\alpha$ have been shown to induce hyper-regenerative mucosal transformation in mouse models. ${ }^{5-8}$ Increased expression of interferon- $\gamma$, tumour necrosis factor- $\alpha$, interleukin-2, and interleukin- 6 has been found at the mRNA or protein level in CD intestinal mucosa and in gluten specific $T$ cell clones derived from intestinal biopsy specimens from CD patients challenged with gluten peptides. ${ }^{9-11}$

The mechanism by which cytokines cause mucosal alterations remains speculative. Recent data indicate that they are important because they induce degradation of extracellular matrix (ECM) in mucosal transformationfor example, the villus atrophy observed shortly after in vivo gluten challenge of patients with CD strongly suggests a loss of ECM. ${ }^{9}{ }^{12}$ This hypothesis is supported by the recent finding that matrix metalloproteinase (MMP)-1 and -3 were upregulated at the mRNA, protein, and functional level in fetal intestine after in vitro stimulation with pokeweed mitogen, and that addition of a synthetic inhibitor of MMPs inhibited pokeweed mitogen induced villus atrophy. ${ }^{13}$ In addition, inhibition of tumour necrosis factor- $\alpha$ using fusion proteins also resulted in a decrease in MMP-1 and -3, together with an improvement in villus atrophy in the same in vitro model. ${ }^{14}$

Cytokines such as interleukin-1 and tumour necrosis factor- $\alpha$ can increase expression of certain MMPs such as MMP-1 and inhibit synthesis of ECM proteins such as collagen I. ${ }^{15-17}$ On the other hand, supernatants of gut lymphocytes from mice with graft versus host

Abbreviations used in this paper: $C D$, coeliac disease; ECM, extracellular matrix; MMP, matrix metalloproteinase; TIMP, tissue inhibitor of metalloproteinases. 
disease stimulated collagen synthesis in cultured fibroblasts. ${ }^{18}$ Investigations of subepithelial collagen from patients with CD produced diverse results. Using van Gieson staining, Bossart et $a l^{19}$ found increased subepithelial collagen deposition in $36 \%$ of CD patients in comparison with controls. In contrast, immunohistochemical examination of collagens type I and III showed no thickening of the subepithelial collagen in mucosal biopsy specimens from four patients with $\mathrm{CD} .^{20}$

MMPs which are central enzymes in the regulation of ECM turnover can be classified according to their substrate specificity: the collagenases, MMP-1, -8 , and -13 , are the most specific proteases, cleaving the triple helix of collagens I, II, and III at a single site. ${ }^{21} 22$ MMP-1 is secreted by several connective tissue cells and macrophages. Increased expression of MMP-1 and MMP-3 mRNA has been shown in the intestine in Crohn's disease and ulcerative colitis. ${ }^{23}{ }^{24}$ The main collagen in the gastrointestinal tract is collagen I, as shown by biochemical analysis and in situ hybridisation in normal intestine. ${ }^{25}{ }^{26} \mathrm{MMP}-3,-10$, and -11 , the stromelysins, constitute the second main group, with broader substrate specificity. MMP-3 also plays a central role in the activation of MMP-1 by proteolysis. ${ }^{21}{ }^{22}$

The expression and activity of MMPs are regulated at several levels. MMPs are secreted as inactive proenzymes. On proteolytic cleavage, the active zinc-containing site is unfolded. Inhibition of MMPs is caused by molecules such as $\alpha_{2}$ macroglobulin and the tissue inhibitors of metalloproteinases (TIMPs). Four members of the TIMP family are known so far, of which TIMP-1 is by far the most predominant and most important representative. ${ }^{27}{ }^{28}$

Since altered ECM metabolism may play a role in the pathogenesis of mucosal atrophy in $\mathrm{CD}$, we studied the changes in mRNA expression of the main fibrillar collagen degrading enzyme, MMP-1, its activator, MMP-3, and their inhibitor, TIMP-1, in duodenal biopsy samples from patients with CD before and after treatment with a gluten free diet as well as in healthy control mucosa using in situ hybridisation. This was compared with collagen-I mRNA expression to investigate whether decreased collagen I synthesis could contribute to the tissue loss in CD.

\section{Materials and methods}

PATIENTS

We studied ten patients with active CD (six women and four men; age 19-71 years; four presented with diarrhoea, weight loss, and anaemia, one with diarrhoea and weight loss, and five were oligosymptomatic showing anaemia and/or a slight increase in stool frequency) and ten patients with irritable bowel syndrome (eight women and two men; age 26-63 years; all presenting with unspecific abdominal discomfort and five with an increased stool frequency, but no weight loss) who had normal endoscopic and laboratory findings and no IgA antibodies to endomysium. Control biopsy specimens showed normal small bowel histology with no increase in intraepithelial lym- phocytes. Formalin fixed paraffin wax embedded duodenal biopsy specimens were taken from the archives of the Institute of Pathology, Free University of Berlin. Diagnosis of CD was confirmed by the ESPGAN criteria including flattening of the villi and an increase in intraepithelial lymphocytes in the initial biopsy specimen and prompt clinical response to a gluten free diet. ${ }^{29}$ In six of the CD patients, control biopsy specimens were obtained after 4-23 months of a gluten free diet; an improvement in villus architecture was observed. All CD patients tested were highly positive for IgA antibodies to endomysium.

PLASMIDS

cDNA probes were prepared by subcloning the cDNA fragments in the run-off transcription vector pGEM1 (Promega Biotech, Madison, Wisconsin, USA) for MMP-1, MMP-3, and procollagen $\alpha 1(\mathrm{I})$ and the vector pAMP1 for TIMP-1. The probe for human TIMP-1 was kindly provided by H-D Orzechowski. ${ }^{30}$ The cDNA probe for human MMP-1 was obtained from ATCC (No 57684, collagenase 1), and that for rat procollagen $\alpha 1(\mathrm{I})$ was kindly provided by Dr Genovese (Farmington, Connecticut, USA). The 600 bp EcoRI/HindIII fragment of pTR1, a gift from Dr L M Matrisian (Nashville, Tennessee, USA), served as a template for the generation of MMP-3 specific RNA probes after subcloning in pGEM1. ${ }^{31}$ The nucleic acid sequences of the probes MMP-1 and MMP-3 were confirmed on a DNA sequencer (model 373A; Applied Biosystems, Foster City, California, USA). The specificity of the probes for procollagen $\alpha 1$ (I) and TIMP-1 was confirmed by restriction endonuclease digestion and partial sequence analysis.

\section{IN SITU HYBRIDISATION}

After linearisation, ${ }^{35} \mathrm{~S}$-labelled run-off antisense and sense (control) transcripts were generated using T7 or SP6 RNA polymerases (Promega-Biotech). In situ hybridisation for the detection of MMP-1, MMP-3, TIMP-1, and procollagen $\alpha 1$ (I) transcripts was performed as described previously. ${ }^{32}$ In brief, dewaxed and rehydrated paraffin sections were exposed to 0.2 $\mathrm{M} \mathrm{HCl}$ and $0.125 \mathrm{mg} / \mathrm{ml}$ Pronase (Boehringer, Mannheim, Germany), followed by acetylation with $0.1 \mathrm{M}$ triethanolamine $(\mathrm{pH} 8.0) / 0.25 \%$ $(\mathrm{v} / \mathrm{v})$ acetic anhydride. Dehydration was achieved through graded ethanols. Slides were hybridised to $2 \times 10^{5}$ to $4 \times 10^{5} \mathrm{cpm}$ labelled probes for 16 hours at $50^{\circ} \mathrm{C}$. Washing and autoradiography were carried out as described previously. ${ }^{32}$ Exposure times were 15 days (procollagen $\alpha 1(\mathrm{I})$ ) and 25 days (MMP-1, MMP-3, and TIMP-1).

QUANTIFICATION AND STATISTICAL ANALYSIS The number of positive cells in the subepithelial region and the lamina propria were counted per high power field at 400-fold magnification (equal to $0.2 \mathrm{~mm}^{2}$ ). For each sample, at least three randomly selected areas were evaluated. Because of patchy epithelial staining for MMP-1 mRNA, at least six high power fields were 

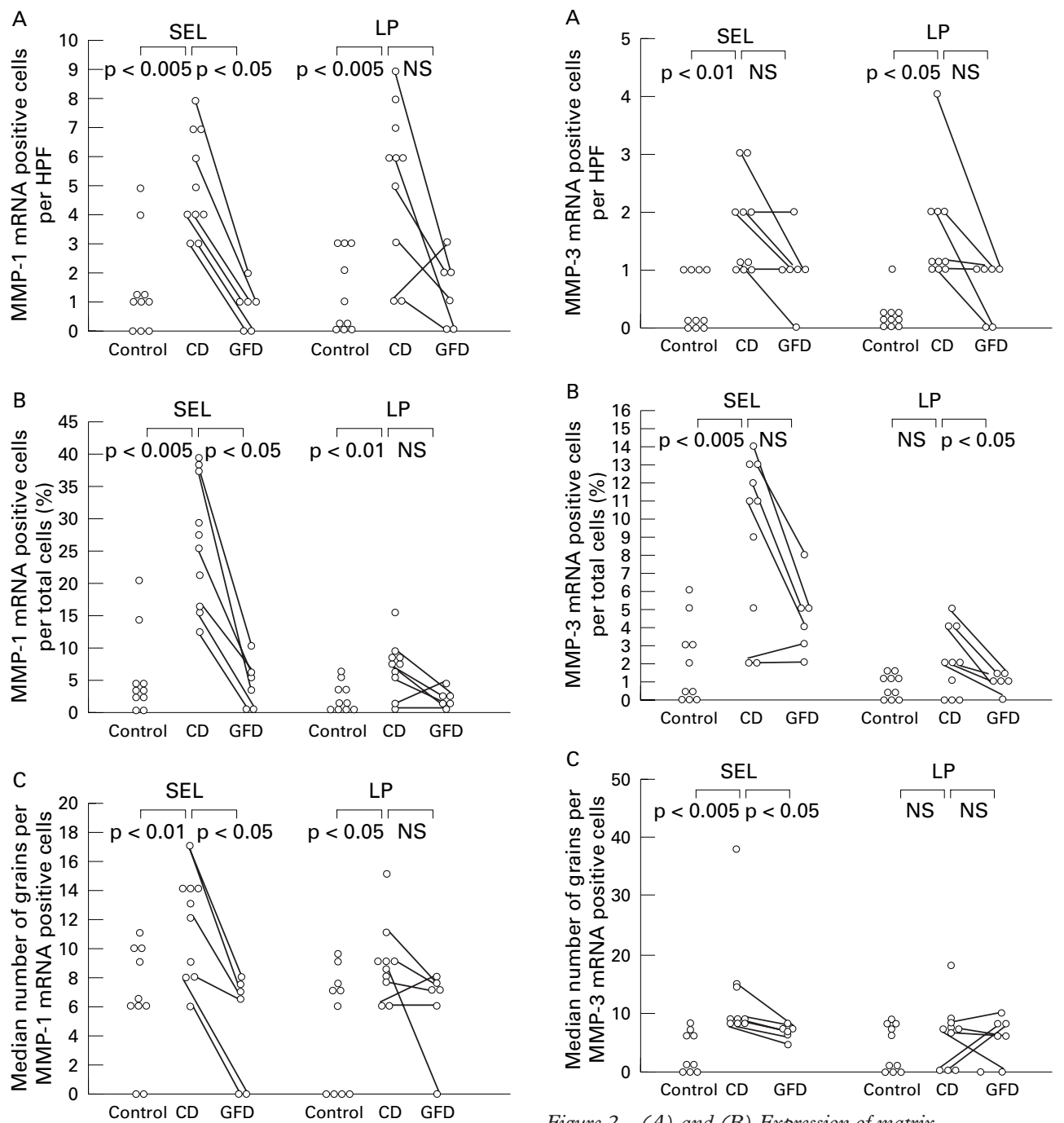

Figure 1 The number (A) and proportion (B) of cells expressing matrix metalloproteinase-1 (MMP-1) $m R N A$ is significantly increased in patients with coeliac disease (CD) compared with the controls, in both the subepithelial layer (SEL) and the lamina propria (LP). A significant reduction in $M M P-1$ transcripts in the subepithelial cell layer was found in biopsy specimens taken from $C D$ patients on a gluten free diet (GFD) in comparison with untreated patients. The number of positive cells in the subepithelial region and the lamina propria were counted per high power field at a 400-fold magnification. (C) $M M P-1 m R N A$ expression per positive cell is also increased in untreated CD compared with controls and compared with after treatment with a gluten free diet. Results are expressed as number of positive cells per high power field $(H P F)(A)$, percentage of positive cells per total number of cells (B), and number of grains per positive cell (C). Results are from 10 patients with CD, 10 controls, and six of the CD patients after treatment with a gluten free diet.

C

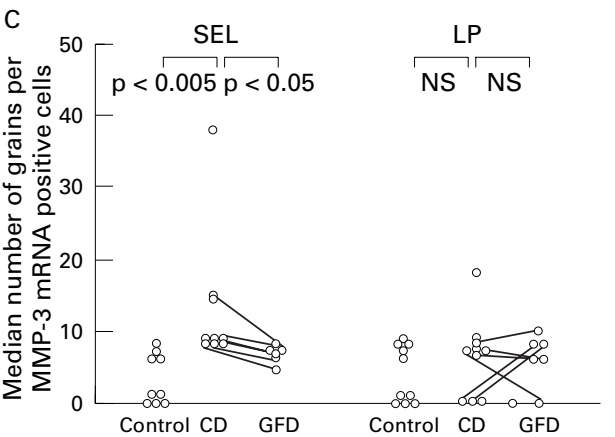

Figure 2 (A) and $(B)$ Expression of matrix metalloproteinase-3 (MMP-3) mRNA is significantly increased in the subepithelial layer (number of positive cells per high power field (HPF) and percentage of total number of cells that were positive) and in the lamina propria in patients with coeliac disease (CD) compared with controls (positive cells per high power field). The reduction in $M M P-3$ transcripts in $C D$ patients after a gluten free diet $(G F D)$ is only significant in the lamina propria (B). (C) In biopsy specimens from untreated $C D$ patients compared with controls and after treatment with a gluten free diet, the $M M P-3 m R N A$ expression per positive cell is increased in the subepithelial layer, but not in the lamina propria. For further explanations, see fig 1.

analysis, median values were compared by the Mann-Whitney U test for unpaired samples (CD $v$ controls) and by the Wilcoxon test for paired samples (CD $v$ CD after gluten free diet) using a standard statistics program (Stat View

counted per sample for epithelial MMP-1 mRNA quantification. Results were expressed as the number of positive cells per high power field or the percentage of the total number of cells that were positive. Control hybridisations with "sense" probes for MMP-1, MMP-3, TIMP-1, and procollagen $\alpha 1$ (I) mRNA showed only weak homogeneous background signal (zero to two grains per cell). Therefore cells expressing more than three times the background signal (six grains or more) were scored positive. mRNA expression per cell was quantified by counting grains above individual positive cells in three high power fields. For statistical 4.51 ${ }^{+}$; Brain Power Inc, Calabasas, California, USA) on a Macintosh PC (Apple Computer Inc, Munich, Germany).

\section{Results}

MMP-1 AND MMP-3

In biopsy specimens from patients with untreated CD there was a significant increase in cells positive for MMP-1 and MMP-3 transcripts per high power field in the subepithelial cell layer compared with controls $(p<0.005$ and $\mathrm{p}<0.01$ respectively; figs 1 and 2). Expression of MMP-1 and MMP-3 mRNA was 

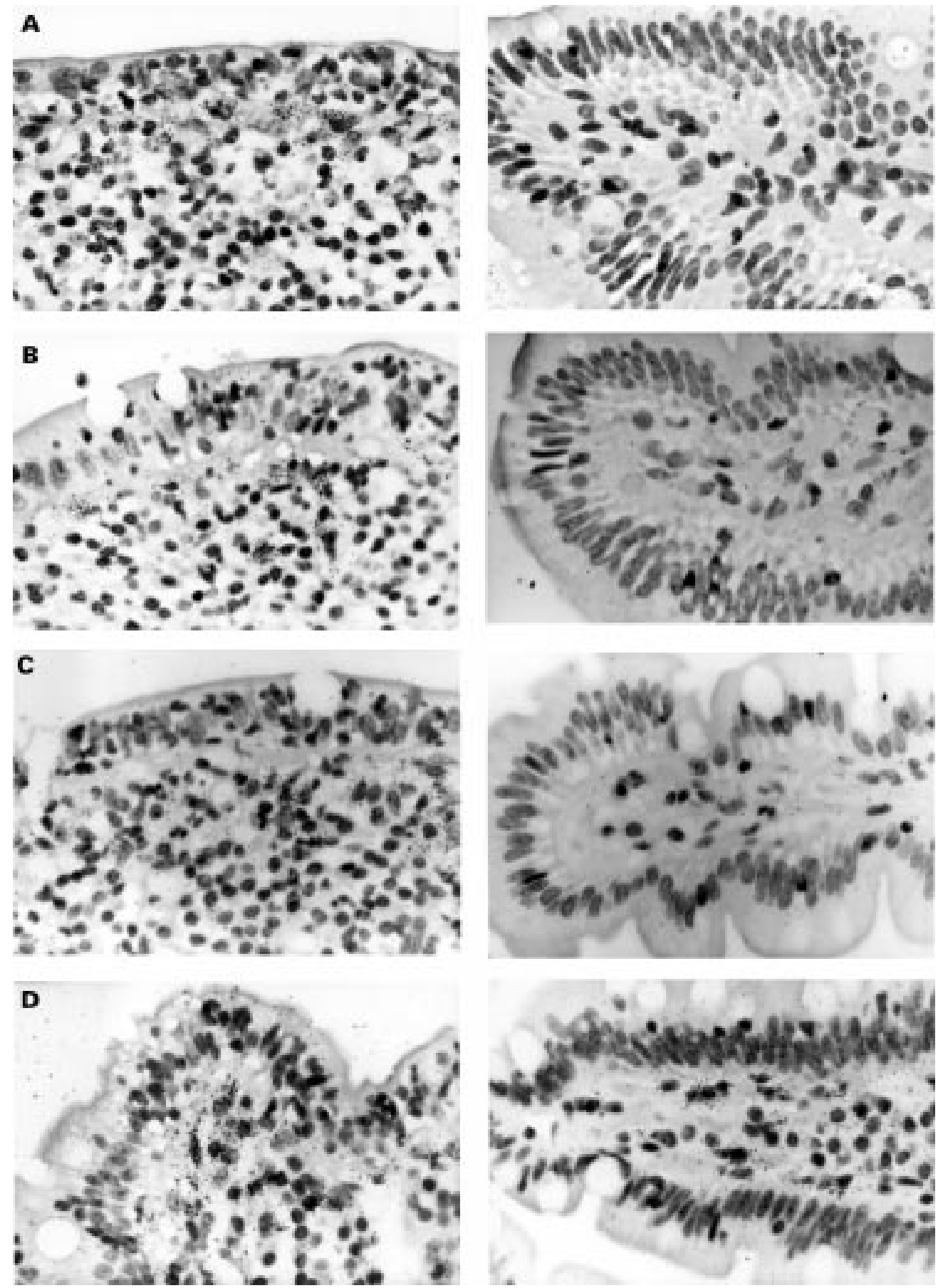

Figure 3 Localisation of matrix metalloproteinase $(M M P)-1(A), M M P-3(B)$, tissue inhibitor of metalloproteinases (TIMP)-1 (C), and collagen I (D) mRNA in the duodenal mucosa in coeliac disease (CD) (left) and uninflamed control tissue (right) by in situ hybridisation. Black (white in dark field photographs in CD) grains indicate hybridisation of RNA antisense probes to $m R N A$ in the tissue. In $C D$, expression of $M M P-1$ and $M M P-3 m R N A$ was found almost exclusively in the subepithelial cell layer and the adjacent region of the lamina propria. MMP-1 and MMP-3 mRNA positive cells were morphologically assessed as macrophages or fibroblasts and myofibroblasts. In addition, expression of MMP-1 mRNA was detected in epithelial cells (E). TIMP-1 mRNA localised to the same regions as MMP-1 and MMP-3 mRNA but without epithelial staining $(C)$. Positive staining for collagen I $m R N A$ can be seen over subepithelial myofibroblasts and fibroblasts (D). (F) Sense hybridisation for procollagen I. Original magnification $\times 100$ (dark field photographs and E) and $\times 200$.

silarly increased in the lamina propria of biopsy specimens from untreated CD patients compared with controls $(\mathrm{p}<0.005$ and $\mathrm{p}<0.05$ respectively). The percentages of positive cells per total counted were higher in untreated CD than controls in both the subepithelial region and the lamina propria for MMP-1 $(p<0.005$ and $\mathrm{p}<0.01$ respectively; fig 1 ), but only in the subepithelial cell layer for MMP-3 $(\mathrm{p}<0.005$; fig 2). After a gluten free diet, MMP-1 and MMP-3 mRNA expression returned to normal levels (figs 1 and 2). However, the decrease in MMP-3 mRNA levels did not reach statistical significance except for total positive cell numbers in the lamina propria. MMP-1 and MMP-3 mRNA positive cells showed large 

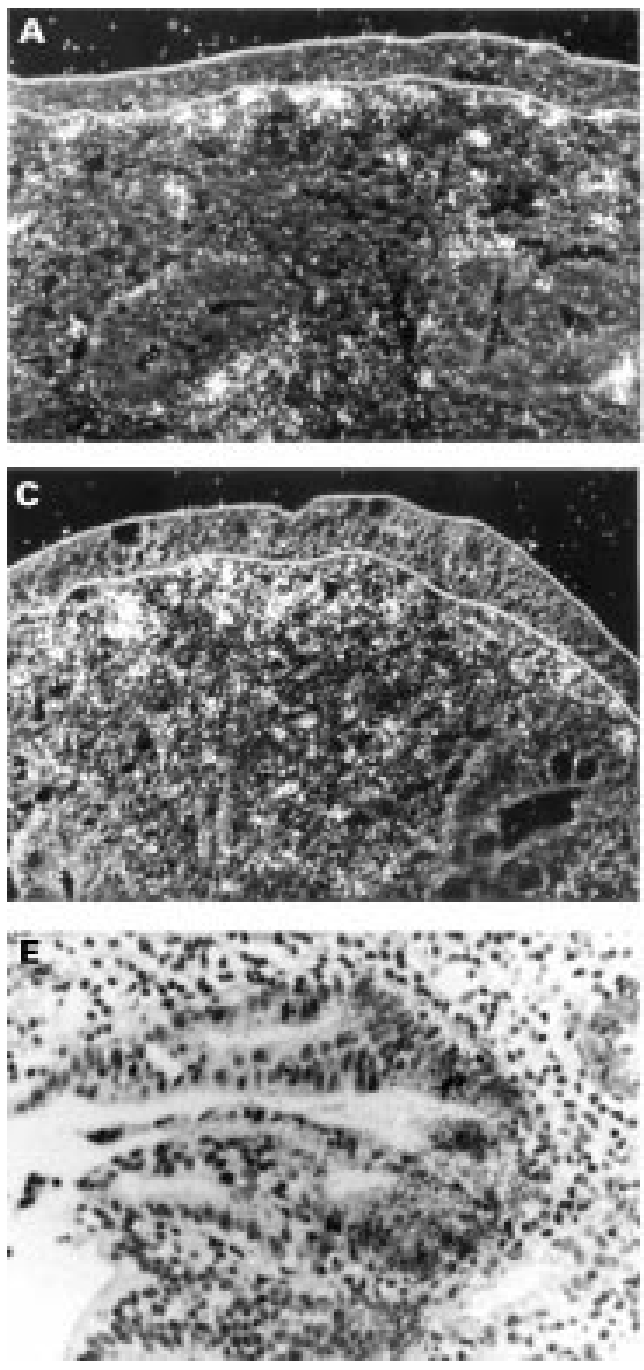

Figure 3-continued

oval nuclei and bright chromatin, thus morphologically resembling fibroblasts, myofibroblasts, and macrophages (fig 3). Apart from fibroblasts, myofibroblasts, and macrophages in the subepithelial region, some pericryptal fibroblasts in the lamina propria displayed positive signals. MMP-1 and MMP-3 mRNA
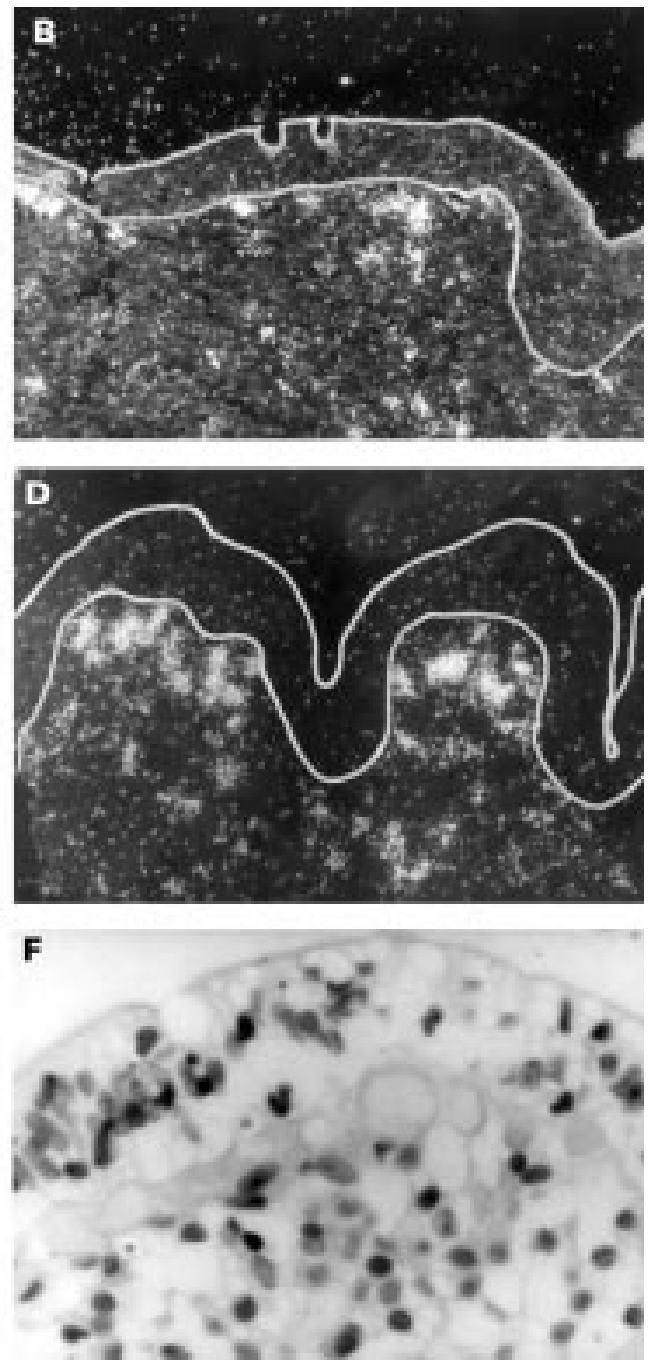

was detected only in the stroma underneath the surface epithelium, and not in the intercryptal stroma.

MMP-1 mRNA expression was also found in epithelial cells and localised to identical regions on serial sections. Epithelial cell expression of MMP-1 mRNA was higher in
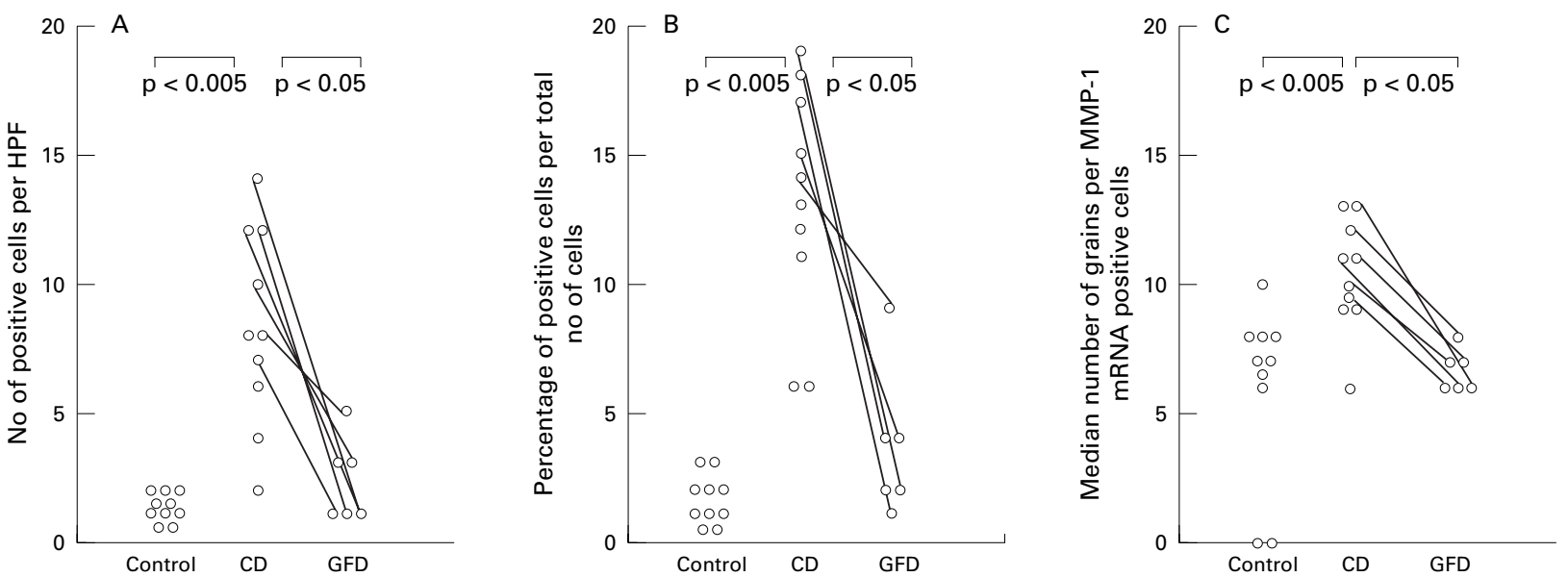

Figure $4(A, B)$ The expression of matrix metalloproteinase (MMP)-1 mRNA in untreated coeliac disease (CD) epithelial cells is significantly increased per high power field and per total cells compared with controls and treated $C D$. (C) MMP-1 mRNA expression per positive cells is also increased in untreated CD compared with controls and treated CD. For further explanations, see fig 1. 

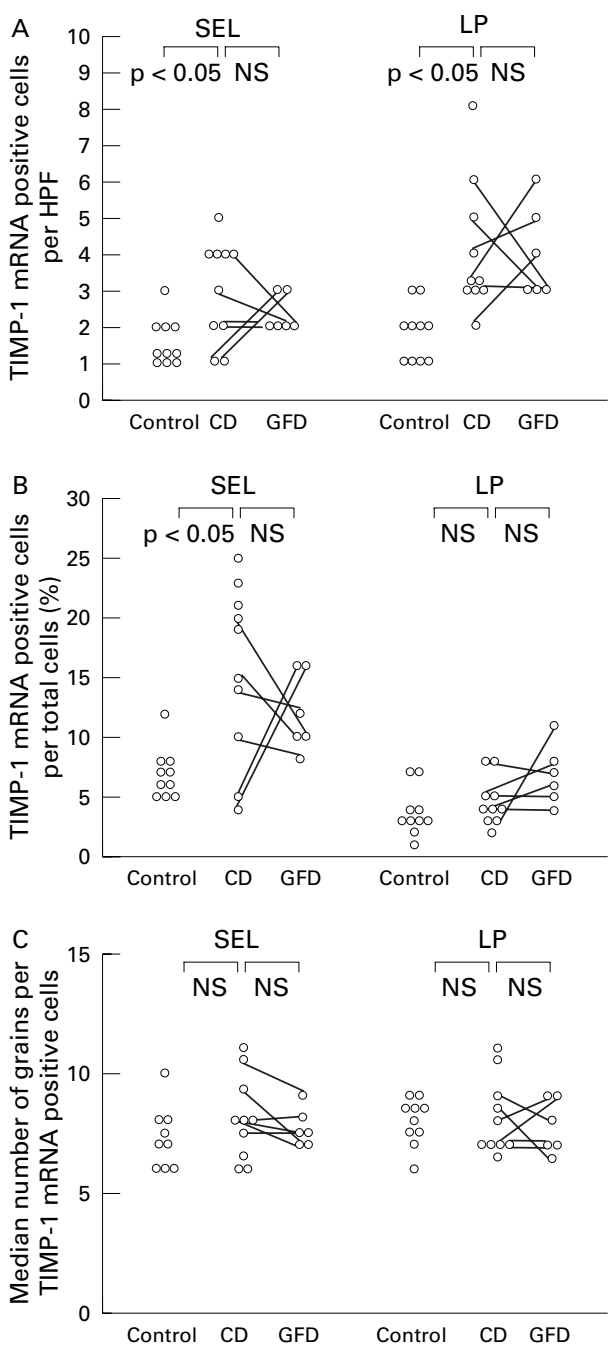

Figure 5 Expression of tissue inhibitor of metalloproteinases (TIMP) $1 \mathrm{mRNA}$ is significantly increased in the subepithelial layer of biopsy specimens from patients with coeliac disease (CD) (as percentage of positive cells per total number of cells (B) as well as positive cells per high power field $(H P F)(A)$ ) and in the lamina propria (A) compared with controls. (C) TIMP-1 mRNA expression is not altered by the gluten free diet in $C D$ biopsy specimens, as TIMP-1 mRNA expression per positive cell is also not increased in untreated $C D$ compared with controls and treated CD. For further explanations, see fig 1 .

biopsy samples from patients with untreated $\mathrm{CD}$ than in controls $(\mathrm{p}<0.005)$ and was decreased after treatment with the gluten free diet $(p<0.05$, figs 3 and 4). In contrast with subepithelial staining, epithelial staining for MMP-1 mRNA was patchy. Intraepithelial lymphocytes were always negative for MMP-1 mRNA. No staining of epithelial cells was seen in slides hybridised with MMP-3 transcripts either in controls or CD.

Not only positive cell numbers but also the intensity of MMP-1 and -3 mRNA expression per cell was increased in untreated $C D$ compared with controls (MMP-1: epithelial, $\mathrm{p}<0.005$; subepithelial, $\mathrm{p}<0.01$; lamina propria, $\mathrm{p}<0.05$; MMP-3: subepithelial, $\mathrm{p}<0.005$; lamina propria, $\mathrm{p}<0.05$; figs 1,2 , and 4 ) and in untreated $\mathrm{CD}$ compared with treated $\mathrm{CD}$ (MMP-1: epithelial, $\mathrm{p}<0.05$; subepithelial, $\mathrm{p}<0.05$; MMP-3: subepithelial, $\mathrm{p}<0.05)$. Cells containing more than 25 grains per cell were
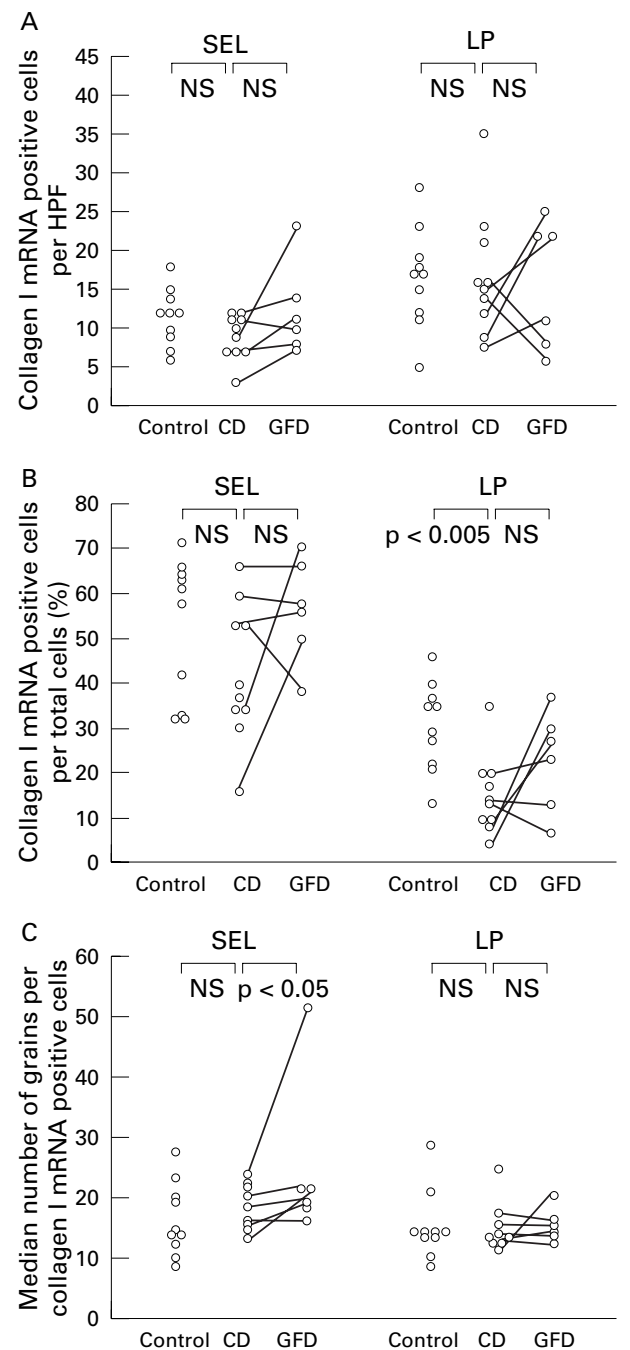

Figure 6 Expression of procollagen a1(I) $m R N A$ in the duodenal mucosa of patients with active and treated CD and in uninflamed control tissue. Only the percentages of procollagen $\alpha 1$ (I) $m R N A$ positive cells per total cells are significantly reduced in CD biopsy specimens compared with controls. After a gluten free diet, procollagen a1(I) $m R N A$ expression per positive cell in the subepithelial layer is increased compared with before the gluten free diet. For further explanations, see fig 1.

detectable in eight of ten CD biopsy specimens before a gluten free diet, but only in one of ten control biopsy specimens and in none of the CD biopsy specimens after the gluten free diet. Median numbers of grains per cell for MMP-1 and -3 mRNA were not much higher in biopsy specimens from untreated CD patients than in those from patients after the gluten free diet, because the number of cells weakly expressing MMP-1 and MMP-3 mRNA were also increased (figs 1 and 2).

TIMP-1

An increase in TIMP-1 mRNA expression was detected in biopsy specimens from untreated CD patients in comparison with controls, showing weak but specific signals in the subepithelial layer and the lamina propria (fig 3). Percentages of cells staining positive for TIMP-1 mRNA were significantly increased only in the subepithelial layer in untreated CD compared with controls (fig 5). Expression of 
TIMP-1 mRNA in CD biopsy specimens was not significantly altered by the gluten free diet (fig 5). TIMP-1 mRNA positive cells also morphologically resembled subepithelial fibroblasts, myofibroblasts, or macrophages. In addition to these three cell types, lymphoid cells of the lamina propria and endothelial cells were positive for TIMP-1 mRNA. No signal for TIMP-1 mRNA was found over epithelial cells or intraepithelial lymphocytes in CD patients or controls. The median numbers of grains per cell were slightly but not significantly higher in biopsy specimens from untreated CD patients compared with controls and compared with the same patients after treatment with a gluten free diet (fig 5).

PROCOLLAGEN $\alpha 1$ (I)

The number of cells expressing procollagen $\alpha 1$ (I) transcripts was the same in biopsy specimens from untreated CD patients and controls when considering positive cell numbers (fig 6 and fig 3). Percentages of cells positive for procollagen $\alpha 1$ (I) mRNA were decreased in biopsy specimens from CD patients not on a gluten free diet compared with controls in the lamina propria $(p<0.005)$, but changes in the subepithelial layer were not significant $(p=0.112$, fig 6 ). When biopsy specimens from patients on a gluten free diet were compared with those from untreated CD patients, there was a trend towards an increased number of collagen I mRNA positive cells in the subepithelial cell layer and an increased expression per cell after treatment with the gluten free diet $(p=0.059$ and $\mathrm{p}<0.05$ respectively, fig 6 ). Procollagen $\alpha 1$ (I) mRNA positive cells resembled subepithelial myofibroblasts and fibroblasts. No specific signals for procollagen $\alpha 1$ (I) mRNA were detected over epithelial cells or intraepithelial lymphocytes.

\section{Discussion}

We investigated mRNA expression of two major ECM degrading enzymes, MMP-1 and MMP-3, their physiological inhibitor, TIMP-1, and the major ECM protein, collagen I, in intestinal biopsy specimens from patients with CD. We found greatly increased expression of MMP-1 mRNA, slightly upregulated expression of MMP-3 and TIMP-1 mRNA, but almost unaltered collagen I mRNA levels in specimens from untreated patients with CD compared with controls. When specimens of untreated patients with CD were compared with those of the same patients after a gluten free diet, a clear decrease in MMP-1 mRNA and a trend towards a lower expression of MMP-3 mRNA were observed, although TIMP-1 mRNA remained increased in comparison with controls. Remarkably, levels of collagen I mRNA were similar in all patients and controls examined. These results suggest a shift of ECM turnover towards ECM degradation in the intestinal mucosa of patients with untreated CD.

In contrast with the findings of Pender et $a l,{ }^{1314}$ who investigated metalloproteinase expression in pokeweed mitogen challenged fetal gut cultures, we found that the expression of
MMP-1 predominated over that of MMP-3. MMP-1 is a key enzyme in the degradation of the interstitial collagens types I and III, which form the backbone of the intestinal ECM. ${ }^{20} 2633$ The rapid collapse of the villus architecture which can occur within hours of challenging CD patients with gliadin in vivo or after the addition of pokeweed mitogen or recombinant MMPs to fetal gut explants is most likely due to degradation of interstitial collagens. ${ }^{9}{ }^{12} 13$ Degradation of the basement membranes would probably have a more delayed effect on intestinal structure. Major tissue destruction can also be brought about by MMP-3, a protease that is capable of degrading several constituents of basement membranes such as collagen type IV, heparan sulphate, proteoglycans, and laminins. ${ }^{21}$ The importance of MMP-3 is underscored by the finding that inhibition of MMP-3, which is a potent activator of MMP-1, is more effective in the prevention of mucosal gut injury than inhibition of MMP-1. ${ }^{13} 142122$ Epithelial cell shedding, as seen in CD, could be the result of preferential basement membrane degradation by MMP-3, since intestinal epithelial cell adherence and differentiation depend on the composition and integrity of the underlying basement membrane. ${ }^{34}$

Fibroblasts, myofibroblasts, and macrophages were found to be the predominant cellular sources of MMP-1 and MMP-3 transcripts. In fact, a recent study shows predominant colocalisation of MMP-3 mRNA with $\alpha$-actin positive intestinal fibroblasts and myofibroblasts. $^{35}$ Stimulation of (myo-)fibroblasts and macrophages may result from activation by interleukin-1, interferon- $\gamma$, or tumour necrosis factor- $\alpha$, which are mainly derived from $\mathrm{T}$ cells and macrophages. ${ }^{91036}$ The location of the ECM degrading enzymes immediately beneath the epithelium could explain the divergence of villus atrophy coupled with elongated crypts in CD. The reason for this may be related to inhomogeneous tissue distribution of activating cytokines.

Surprisingly, we also found epithelial cells to express mRNA for MMP-1 in CD intestinal mucosa. Epithelial staining was virtually absent on intestinal samples from patients with ulcerative colitis, Crohn's disease, controls, or CD patients on a gluten free diet (unpublished results). ${ }^{23}{ }^{24}$ On serial sections, expression of epithelial MMP-1 mRNA was always found in the same locations and no staining was observed on epithelial cells with the sense probe. It is therefore unlikely that epithelial MMP-1 expression in CD is due to unspecific probe hybridisation to epithelial cells. In fact, synthesis of MMP-1 mRNA has been shown in a rat epithelial cell line and in epithelial cells of bile ducts of cirrhotic liver. ${ }^{37}{ }^{38}$ The epithelial expression of MMP-1 mRNA exclusively in untreated CD may suggest a distinct state of activation of epithelial cells in this disease, probably caused by a distinct profile of inflammatory mediators.

Although present in controls, CD biopsy specimens showed significantly higher expression of TIMP-1 mRNA, but this increase was much less pronounced than that seen for 
MMP-1. MMP activity is tightly regulated by TIMPs, which are often synthesised by the same cells that synthesise MMPs or by neighbouring cells. Both MMP-1 and TIMP-1 expression are upregulated by factors such as epidermal growth factor, interleukin-1, and platelet derived growth factor. ${ }^{31}{ }^{39}$ In accordance with these findings, we found TIMP-1 mRNA in fibroblasts and macrophages, cells that were also found to contain MMP-1 and MMP-3 mRNA. In contrast with MMP-1 and MMP-3, TIMP-1 mRNA expression in CD was not affected by the gluten free diet (fig 5). This may reflect continual increased expression of inducing cytokines such as epidermal growth factor during repair. The sustained increased expression of TIMP-1 in treated CD argues in favour of increased MMP expression and against decreased TIMP-1 expression as being responsible for villus atrophy.

Collagen synthesis and deposition are mainly controlled at the mRNA level and by proteolysis. For example, parallel changes in collagen mRNA and protein levels have been shown by Graham et $a l^{16}$ in human intestinal smooth muscle cell cultures. In vivo, enhanced synthesis of collagen or expression of collagen mRNA have been shown in a murine graft versus host disease model of intestinal inflammation and in intestinal biopsy specimens in Crohn's disease and ulcerative colitis. ${ }^{1826} \mathrm{We}$ found no evidence of increased collagen I synthesis in active CD biopsy specimens, and percentages of procollagen $\alpha 1$ (I) mRNA positive cells were even decreased in the lamina propria of duodenal biopsy specimens from untreated CD patients compared with controls. This decrease is apparently due to the increased cellular infiltration with immune cells in specimens from untreated CD patients. There may be different turnover rates for other molecules of the ECM, as Colombel et $a l^{40}$ found an increased rate of hyaluronic acid turnover, but normal levels of procollagen III N-terminal peptide in jejunal perfusion fluid from CD patients in comparison with normal controls, which also argues against altered collagen metabolism in CD. Thus the spectrum of cytokines expressed in the murine graft versus host disease animal model or in chronic inflammatory bowel diseases appears to induce the synthesis of collagen, whereas a distinct cytokine pattern in CD may not alter collagen $\alpha 1$ (I) mRNA expression.

Although higher levels of MMP-1 and MMP-3 transcripts in CD before a gluten free diet suggest enhanced ECM degradation, activation of MMP-1 and MMP-3 occurs only after proteolytic processing with exposure of the active centre. Full activity of MMP-1 is dependent on further cleavage by MMP-3. In addition, TIMPs tightly regulate the activated enzymes. $^{21}{ }^{22}$ Thus a surplus of TIMP-1 could neutralise increased MMP activity. However, in our study TIMP-1 mRNA expression remained high after treatment with the gluten free diet, indicating an imbalance in favour of MMP-1 and MMP-3 in untreated CD. MMP activity immediately after gliadin challenge in CD patients may be even higher than in the steady state of untreated CD patients. Although mRNA levels of MMPs do not necessarily reflect enzyme activity, our finding of enhanced MMP-1 and MMP-3 mRNA expression in biopsy specimens from CD patients before the gluten free diet compared with specimens taken after the gluten free diet, in the light of unchanged mRNA levels of TIMP-1 and procollagen I, strongly suggest a central role for the enhanced expression as well as functional activity of MMP-1 and MMP-3 in the pathogenesis of mucosal atrophy observed in $\mathrm{CD}$.

In summary, biopsy specimens from CD patients not on a gluten free diet showed increased expression of MMP-1 and MMP-3 mRNA, which was mainly located in subepithelial fibroblasts, myofibroblasts, and macrophages. The decreased ratio of cells expressing collagen I and TIMP-1 mRNA to those expressing MMP-1 and MMP-3 mRNA in untreated CD suggests a shift towards ECM degradation. ECM degradation by activated subepithelial fibroblasts, myofibroblasts, and macrophages may be an important mechanism of mucosal transformation in CD.

This work was supported by the Deutsche Forschungsgemeinschaft Da $102 / 2-1$

1 Marsh MN. The morphology and immunopathology of the jejunal lesion in gluten-sensitivity. Eur f Gastroenterol Hepatol 1991;3:163-8.

2 Halstensen TS, Brandtzaeg P. Activated T lymphocytes in the celiac lesion: non-proliferative activation (CD25) of $\mathrm{CD} 4+\alpha / \beta$ cells in the lamina propria but proliferation $(\mathrm{Ki}-$ $67)$ of $\alpha / \beta$ and $\gamma / \delta$ cells in the epithelium. Eur $\mathcal{F}$ Immunol 1993;23:505-10.

3 Lundin KEA, Scott H, Hansen T, et al. Gliadin-specific, HLA-DQ $\left(\alpha 1^{\star} 0501, \beta 1^{\star} 0201\right)$ restricted $\mathrm{T}$ cells isolated from the small intestinal mucosa of celiac disease patients. f Exp Med 1993;178:187-96.

4 MacDonald TT, Spencer J. Evidence that activated mucosal $T$ cells play a role in the pathogenesis of enteropathy in human small intestine. $\mathcal{F}$ Exp Med 1988;167:1341-9.

5 Garside P, Felstein V, Green EA, et al. The role of interferon $\alpha / \beta$ in the induction of intestinal pathology in mice. Immunology 1991;74:279-83.

6 Mowat AM. Antibodies to IFN- $\gamma$ prevent immunologically mowated intestinal damage in murine graft versus host
mediated mediated intestinal damage in murin

7 Mowat AM, Hutton AK, Garside P, et al. A role for Mowat AM, Hutton AK, Garside $\mathrm{P}$, et al. A role for
interleukin-1 $\alpha$ in immunologically mediated intestinal pathology. Immunology 1993;80:110-15.

8 Piguet P-F, Grau GE, Allet B, et al. Tumor necrosis factor/ cachectin is an effector of skin and gut lesions of the acut phase of graft-vs.-host disease. $\mathcal{F} \operatorname{Exp}$ Med 1987;166:1280-

9 Kontakou M, Przemioslo RT, Sturgess RP, et al. Cytokine mRNA expression in the mucosa of treated coeliac patients after wheat peptide challenge. Gut 1995;37:52-7.

10 Przemioslo RT, Kontakou M, Nobili V, et al. Raised proinflammatory cytokines interleukin 6 and tumour necrosis factor $\alpha$ in coeliac disease mucosa detected by immunohistochemistry. Gut 1994;35:1398-403.

11 Nilsen EM, Lundin KEA, Krajci P, et al. Gluten specific, HLA-DQ restricted $T$ cells from coeliac mucosa produce cytokines with Th1 or Th0 profile dominated by interferon r. Gut 1995;37:766-76.

12 Marsh MN, Loft D, Garner V, et al. Time/dose responses of coeliac mucosae to graded oral challenges with Frazer's fraction III of gliadin. Eur f Gastroenterol Hepatol 1992;4: fraction

13 Pender SL, Tickle SP, Docherty AJ, et al. A major role for matrix metalloproteinases in $\mathrm{T}$ cell injury in the gut. $\mathcal{F}$ Immunol 1997;158:1582-90.

14 Pender SLF, Fell JME, Chamow SM, et al. A p55 receptor mmunoadhesin prevents $\mathrm{T}$ cell-mediated intestinal injury by inhibiting matrix metalloproteinase production. $f$ Immunol 1998;160:4098-103

15 Mauviel A. Cytokine regulation of metalloproteinase gene expression. F Cell Biochem 1993;53:288-95.

16 Graham $\mathrm{M}$, Willey A, Adams J, et al. Interleukin- $1 \beta$ downregulates collagen and augments collagenase expression in human intestinal smooth muscle cells. Gastroenterology 1996;110:344-50.

17 Dayer JM, Beutler B, Cerami A. Cachectin/tumor necrosis factor stimulates collagenase and prostaglandin E2 production by human synovial cells and dermal fibroblasts. F Exp Med 1985;162:2163-8. 
18 DeClerck Y, Draper V, Parkman R. Clonal analysis of murine graft-vs-host disease. II. Leukokines that stimulate host disease. F Immunol 1986;136:3549-52.

19 Bossart R, Henry K, Booth CC, et al. Subepithelial collagen Bossart R, Henry K, Booth CC, et al. Subepitheli
in intestinal malabsorption. Gut 1975;16:18-22.

20 Hein J, Hahn E, Riecken EO. Kollagentypen und mononukleäre Zellen in der Dünndarmschleimhaut: Lokalisation bei Gesunden und Patienten mit Sprue. Verhandlungen de Deutschen Gesellschaft fur Innere Medizin 1977;83:452-5.

21 Birkedal-Hansen H. Proteolytic remodeling of extracellular matrix. Curr Opin Cell Biol 1995;7:728-35.

22 Woessner JF. Matrix metalloproteinases and their inhibitors in connective tissue remodeling. FASEB $\mathcal{F}$ 1991;5:214554.

23 Matthes H, Stallmach A, Matthes B, et al. Hinweise für einen differenten Kollagenmetabolismus bei Morbus Crohn und Colitis ulcerosa. Med Klin 1993;88:185-92.

24 Saarialho-Kere UK, Vaalamo M, Puolakkainen P, et al. Enhanced expression of matrilysin, collagenase, and Enhanced expression of matrilysin, collagenase, and
stromelysin-1 in gastrointestinal ulcers. Am f Pathol 1997; 148:519-26.

25 Graham M, Diegelmann R, Elson C, et al. Collagen content and types in the intestinal strictures of Crohn's disease. Gastroenterology 1988;94:257-65.

26 Matthes H, Herbst H, Schuppan D, et al. Cellular localization of procollagen gene transcripts in inflammatory bowe diseases. Gastroenterology 1992;102:431-42.

27 Matrisian LM. Metalloproteinases and their inhibitors in matrix remodeling. Trends Genet 1990;6:121-5.

28 Goldberg GI Marmer BL, Grant GA, et al. Human 72-kilodalton type IV collagenase forms a complex with a tissue inhibitor of metalloproteases designated TIMP-2. Proc Natl Acad Sci USA 1989;86:8207-11.

29 Walker-Smith JA, Guandalini S, Schmitz J, et al. Revised criteria for diagnosis of coeliac disease. Arch Dis Child 1990;65:909-11.
30 Herbst $\mathrm{H}$, Wege $\mathrm{T}$, Milani $\mathrm{S}$, et al. Tissue inhibitor of metalloproteinase-1 and -2 RNA expression in rat and metalloproteinase-1 and -2 RNA expression in rat
human liver fibrosis. Am $\mathcal{F}$ Pathol 1997;150:1647-59.

31 Matrisian LM, Glaichenhaus N, Gesnel MC, et al. Epidermal growth factor and oncogenes induce transcription of the same cellular mRNA in rat fibroblasts. EMBO $f$ $1985 ; 4: 1435-40$

32 Milani S, Herbst H, Schuppan D, et al. In situ hybridization for collagen types I, III and IV mRNA in normal and fibrotic rat liver. Evidence for predominant expression in non-parenchymal liver cells. Hepatology 1989;10:84-92.

33 Schuppan D, Riecken EO. Molecules of the extracellular matrix: potential role of collagens and glycoprote

34 Hahn U, Stallmach A, Hahn EG, et al. Basement membrane components are potent promoters of rat intestinal epithelial cell differentiation in vitro. Gastroenterology 1990;98:322-5.

35 Pender SLF, Breese E, Günther U, et al. Suppression of T cell mediated injury in human gut by interleukin-10: role of matrix metalloproteinases. Gastroenterology 1998;115: matrix

36 Gifford GE, Flick DA. Tumour necrosis factor. Microbiol Sci 1988;5:104-7.

37 Whitelock JM, Paine ML, Gibbins JR, et al. Multiple levels of post-transcriptional regulation of collagenase (matrix metalloproteinase 1) in an epithelial cell line. Immunol Cell Biol 1993;71:39-47.

38 Milani S, Herbst H, Schuppan D, et al. Differential expression of matrix-metalloproteinase- 1 and -2 genes in norm and fibrotic human liver. Am f Pathol 1994;144:528-37.

39 Murphy G, Reynolds HH, Werb Z. Biosynthesis of tissue inhibitor of metalloproteinases by human fibroblasts in culture. F Biol Chem 1985;260:3079-83.

40 Colombel JF, Hallgren R, Engstrom-Laurent A, et al. Hyaluronic acid and type II procollagen peptide in jejunal perfusion fluid as markers of connective tissue turnover. Gastroenterology 1989;96:68-73. 